\title{
Screening of Metal Modified HKUST-1 to Enhance Mercury Removal Efficiency
}

\author{
Mingjie Zhang ${ }^{1,2}$, Hongzhe $\mathrm{Li}^{1,2}$, Gang Yang ${ }^{2,3}$, Jiahui $\mathrm{Yu}^{2,3}$, Yipei Chen ${ }^{2,3 *}$ \\ ${ }^{1}$ College of Environmental and Chemical Engineering, Shanghai University of Electric power, 200090 Shanghai, China \\ ${ }^{2}$ New Materials Institute, The University of Nottingham Ningbo China, 315100 Ningbo, China \\ ${ }^{3}$ Key Laboratory for Carbonaceous Wastes Processing and Process Intensification of Zhejiang Province, The University of Nottingham \\ Ningbo China, Ningbo 315100, China.
}

\begin{abstract}
In this work, HKUST-1 and different metal modified HKUST-1 materials were investigated in $\mathrm{Hg}^{0}$ removal performance under air condition. Results showed that the pristine HKUST-1 only has a mercury removal rate of around $20 \%$. After metal modification of HKUST-1, the removal efficiency was significantly improved. Among different modification materials employed in this study, HKUST-1 modified by manganese chloride exhibited the highest $\mathrm{Hg}^{0}$ removal efficiency of $95 \%$ at $250{ }^{\circ} \mathrm{C}$. This value is relatively high compared with most previously reported catalysts. In addition, this study reveals that the enhanced $\mathrm{Hg}^{0} \mathrm{removal}$ efficiency results from the increase of Brønsted acid sites on the sample surface, which is vital to $\mathrm{Hg}^{0}$ removal. Furthermore, it is found that $\mathrm{SO}_{2}$ produced little toxicity to $\mathrm{Mn}(\mathrm{Cl}) / \mathrm{HKUST}-1$ in the presence of $\mathrm{O}_{2}$. This is beneficial to remove $\mathrm{Hg}^{0}$ in the industrial flue gas, where $\mathrm{SO}_{2}$ is a common component. Therefore, $\mathrm{Mn}(\mathrm{Cl}) / \mathrm{HKUST}-1$ synthesized here is a promising catalyst for $\mathrm{Hg}^{0}$ removal.
\end{abstract}

\section{Introduction}

Mercury control has become increasingly important because mercury is a poisonous material that results in a detrimental impact on ecosystems and human beings [1]. Countries are beginning to develop documents to control mercury emissions. In 2015, China began to implement pollutant emission standards (GB13223-2011) [2], which put forward the mercury emission limit of $0.03 \mathrm{mg} / \mathrm{m}^{3}$. Mercury is mainly divided into three forms: particlebound mercury $\left(\mathrm{Hg}^{\mathrm{p}}\right)$, gaseous divalent mercury $\left(\mathrm{Hg}^{2+}\right)$ and elemental mercury $\left(\mathrm{Hg}^{0}\right)$ [3]. Among these, $\mathrm{Hg}^{2+}$ can be removed by wet flue gas desulfurization device because of its high-water solubility. The electrostatic dust removal equipment can be used to remove $\mathrm{Hg}^{\mathrm{p}}$, while $\mathrm{Hg}^{0}$ exists in a stable form and persist for a long time due to its high volatility and low solubility [4]. $\mathrm{Hg}^{0}$ affects the growth of crops and contaminates aquatic life, it can also poison the brain and digestive tract when entering human body [5]. Therefore, there is a considerable interest in developing a novel catalyst to control the emission of elemental mercury $\left(\mathrm{Hg}^{0}\right)$.

Many studies have indicated that a new material, metal organic framework material (MOF), has a good prospect of mercury removal due to its high porosity, large specific surface area and diverse structural morphology $[6,7]$. HKUST-1 is a copper-based MOF material with each $\mathrm{Cu}^{2+}$ binds to a water molecule. The water molecules can be easily removed and thus generating open metal sites. Moreover, HKUST-1 shows a lot of acid sites on the surface which is beneficial to $\mathrm{Hg}^{0}$ adsorption and catalytic oxidation $[8,9]$. Chen et al. found that HKUST-1 exhibited superior $\mathrm{Hg}^{0}$ removal efficiency when adding $\mathrm{HCl}$ into the gas [9]. However, $\mathrm{HCl}$ was proved to be toxic and corrosive. Additionally, many researchers have reported that metal oxides like $\mathrm{CeO}_{\mathrm{x}}$ and $\mathrm{MnO}_{\mathrm{x}}$ et al. have excellent performance on $\mathrm{Hg}^{0}$ removal [10]. Therefore, it is speculated that modification by metal or metal oxides on HKUST-1 can improve the $\mathrm{Hg}^{\circ}$ removal performance. Furthermore, there is a certain amount of $\mathrm{SO}_{\mathrm{x}}$ existed in the coal-fired flue gas and $\mathrm{SO}_{2}$ is the major component. It is difficult for air pollution equipment (APCDs) to completely remove $\mathrm{SO}_{2}$. Many reports demonstrated that $\mathrm{SO}_{2}$ played a negative role in $\mathrm{Hg}^{0}$ removal. Hence, the effect of $\mathrm{SO}_{2}$ on $\mathrm{Hg}^{0}$ removal is significant to study.

In this work, different metal modified HKUST-1 composites were prepared. $\mathrm{MnCl} 2$ was found to to be the optimal modification agent for HKUST-1 in terms of $\mathrm{Hg}^{0}$ removal performance. Moreover, the $\mathrm{SO}_{2}$ effect on the $\mathrm{Hg}^{0}$ removal when using the optimal was also investigated.

\section{Materials and Methods}

All chemicals were purchased from Sinopharm and used directly without any treatment.

\subsection{Preparation of samples}

HKUST-1 powder preparation

The preparation of HKUST-1 was carried out by hydrothermal method as described in Ref [11]. Copper nitrate $\left(\mathrm{Cu}\left(\mathrm{NO}_{3}\right)_{2} \cdot 3 \mathrm{H}_{2} \mathrm{O}, 7.5 \mathrm{~g}\right)$ and trimetric (TMA, $4 \mathrm{~g}$ )

* Corresponding author: z2019011@nottingham.edu.cn. 
were first dissolved in ethanol. The mixed solution was heated under stirring at $50{ }^{\circ} \mathrm{C}$ for $3 \mathrm{~h}$. The mixture was then centrifuged to separate the solid. The solid was placed in an oven at $120^{\circ} \mathrm{C}$ overnight. The dried powder was then washed by methanol to remove impurities. The final product was obtained after centrifugation and drying.

HKUST- $1 / \mathrm{Al}_{2} \mathrm{O}_{3}$ preparation

A certain amount of $\mathrm{Cu}\left(\mathrm{NO}_{3}\right)_{2} \cdot 3 \mathrm{H}_{2} \mathrm{O}$ was dissolved in ethanol and impregnated on $\mathrm{Al}_{2} \mathrm{O}_{3}$ by incipient wetness impregnation method. It was heated at $50{ }^{\circ} \mathrm{C}$ for $3 \mathrm{~h}$. Then, TMA was excessive impregnated on the product and the reaction was heated at $50{ }^{\circ} \mathrm{C}$ for another $3 \mathrm{~h}$. Finally, the sample (HKUST- $1 / \mathrm{Al}_{2} \mathrm{O}_{3}$ ) was washed by methanol and dried at $120^{\circ} \mathrm{C}$.

\section{A/HKUST-1 preparation}

A metal salt with a mass fraction of $10 \%$ was dissolved in ethanol and deionized water $(40 \mathrm{~mL}, 1: 1)$. About $1 \mathrm{~g}$ of HKUST-1 was mixed into the solution. The mixture was stirred at room temperature and ambient pressure for $6 \mathrm{~h}$. After drying at $100{ }^{\circ} \mathrm{C}$ overnight, the solid was calcined at $250{ }^{\circ} \mathrm{C}$ for $2 \mathrm{~h}$ under $\mathrm{N}_{2}$ atmosphere. The samples referred in the following were denoted as HKUST-1 and A/HKUST-1, respectively. (A was europium nitrate, cerium nitrate, manganese nitrate, manganese acetate or manganese chloride).

\subsection{Characterization of the samples}

The structure of the solid phase was examined by powder X-ray diffraction (XRD SmartLab3KW) with a scanning rate of $0.02^{\circ} / \mathrm{s}$. The scanning range is $5-40^{\circ}$. The temperature-programmed reduction with $\mathrm{H}_{2} / \mathrm{He}$ test was conducted by a chemisorption analyser $\left(\mathrm{H}_{2}-\mathrm{TPR}\right.$ Auto Chem I| 2920). Around $0.05 \mathrm{~g}$ sample was used for each test. The test temperature was set from $50-500{ }^{\circ} \mathrm{C}$ at the heating rate of $10{ }^{\circ} \mathrm{C} / \mathrm{min}$ in the flow of $2.5 \% \mathrm{H}_{2}$ in $\mathrm{He}$. Thermal stability test was conducted by using the thermogravimetric analyser (TGA NETZSCH STA49 F3) under nitrogen atmosphere, and the temperature was heated from $30{ }^{\circ} \mathrm{C}$ to $600{ }^{\circ} \mathrm{C}$ at $10^{\circ} \mathrm{C} / \mathrm{min}$ [12]. In-situ diffuse reflectance infrared Fourier transform spectroscopy (in-situ DRIFT VERTEX 70) was adopted to study the acid sites on the surface, with $\mathrm{NH}_{3}$ as the probe molecule [13].

\section{$2.3 \mathrm{Hg}^{0}$ catalytic oxidation performance evaluation}

The $\mathrm{Hg}^{0}$ removal performance evaluation device consists of a Tekran $3300 \mathrm{Hg}^{0}$ continuous monitoring module, an elemental $\mathrm{Hg}^{0}$ calibration module (3310), a dilution probe controller (3340), a sample controller (3320) and a fixed bed quartz $(\mathrm{D}=12 \mathrm{~mm})$. Teflon pipelines and quartz reactors were selected to avoid mercury adsorbed to the equipment. The reaction temperature range is $30-300{ }^{\circ} \mathrm{C}$ with a heating rate of $2^{\circ} \mathrm{C} / \mathrm{min}$. The $\mathrm{Hg}^{0}$ removal efficiency was calculated by the following equation:

$$
\mathrm{E}=\frac{\left[\mathrm{Hg}^{0}\right]_{\mathrm{in}}-\left[\mathrm{Hg}^{0}\right]_{\text {out }}}{\left[\mathrm{Hg}^{0}\right]_{\mathrm{in}}} \times 100 \%
$$

\section{Results and discussion}

\subsection{Activity comparison of HKUST-1 and HKUST- $1 / \mathrm{Al}_{2} \mathrm{O}_{3}$}

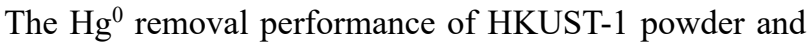
HKUST- $1 / \mathrm{Al}_{2} \mathrm{O}_{3}$ with the same amount of HKUST-1 were conducted. The concentration of $\mathrm{Hg}^{0}$ source was $30 \mu \mathrm{g} / \mathrm{m}^{3}$, and $1500 \mathrm{ml} / \mathrm{min}$ flow rate of air was injected into the reactor. The comparison results were shown in Fig. 1. Before $112{ }^{\circ} \mathrm{C}$, the $\mathrm{Hg}^{0}$ removal efficiency of powder HKUST-1 was lower than that of HKUST- $1 / \mathrm{Al}_{2} \mathrm{O}_{3}$. This can be attributed to the distribution of HKUST-1 was more uniform than the powder. As the temperature continued to increase, the removal efficiency of powder HKUST-1 gradually increased, which reached the highest value of $31.15 \%$ at around $175^{\circ} \mathrm{C}$. Then the removal rate decreased gradually with the increasing temperature and kept stable between $250-300{ }^{\circ} \mathrm{C}$. However, the removal efficiency of HKUST- $1 / \mathrm{Al}_{2} \mathrm{O}_{3}$ dramatically declined after $112^{\circ} \mathrm{C}$ and became zero at $150^{\circ} \mathrm{C}$. This is probably caused by the structure decomposition of HKUST-1 supported on $\mathrm{Al}_{2} \mathrm{O}_{3}$ under this reaction condition. This matches with its colour change observed from light blue to grey, which was not found for HKUST-1 powder.

During the $\mathrm{Hg}^{0}$ removal process for HKUST-1 powder, both adsorption and catalytic oxidation of $\mathrm{Hg}^{0}$ occurred. The oxidation reaction rate rose with the temperature increasing, while the adsorption rate decreased. According to the curve shown in Fig. 1, it can be inferred that the oxidation reaction dominates before $175^{\circ} \mathrm{C}$. However, due to the limited adsorption capacity of HKUST-1, as well as the dropped adsorption rate at high temperature $\left(>175^{\circ} \mathrm{C}\right)$, the whole $\mathrm{Hg}^{0}$ removal efficiency obviously went down.

By comparing the results for two materials, the conclusion can be drawn that HKUST-1 powder was superior to HKUST-1/ $\mathrm{Al}_{2} \mathrm{O}_{3}$ for $\mathrm{Hg}^{0}$ removal performance. Therefore, powder HKUST-1 was selected as the pristine sample for the later modification.

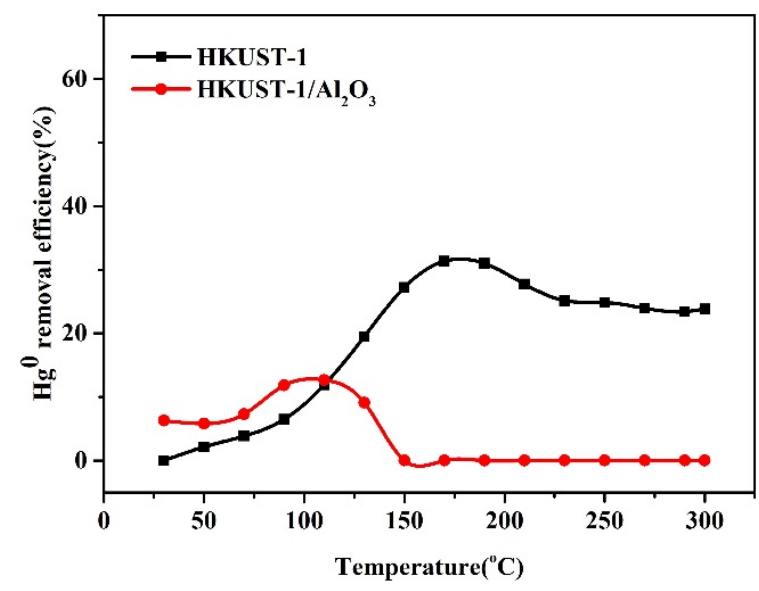

Figure 1 Dynamic activity test trend of prepared samples (Reaction condition: $\mathrm{Hg}^{0}=30 \mu \mathrm{g} / \mathrm{m}^{3}$, GHSV $=1.74 \times 10^{5} \mathrm{~h}^{-1}$, Temperature $30-300^{\circ} \mathrm{C}$.) 


\subsection{Modified HKUST-1 for $\mathrm{Hg}^{0}$ removal test}

Different metal salts were used to modify HKUST-1 and the $\mathrm{Hg}^{0}$ removal results were displayed in Fig. 2(a). It can be seen that $\mathrm{Hg}^{0}$ removal efficiencies of the samples modified are all higher than that of the sample without modification. When the temperature is higher than $200{ }^{\circ} \mathrm{C}$, the high-to-low order of mercury removal rate is as follows: Mn/ HKUST-1 > Ce/HKUST-1 > Eu/HKUST-1 > HKUST-1. The material modified by manganese nitrate exhibited the highest mercury removal efficiency, reaching about $65 \%$. This indicates that $\mathrm{Mn}$ is more helpful to improve the efficiency of $\mathrm{Hg}^{0}$ removal compared to the other metals. Many previous literatures have reported that Mn-based catalyst is one of the best candidate catalysts for the removal of $\mathrm{Hg}^{0}$ [14]. Therefore, the $\mathrm{Hg}^{0}$ removal performance of manganese modified HKUST-1 was mainly studied.

The precursor is one of the important factors affecting the efficiency of adsorbent/catalyst. In this section, manganese nitrate, manganese acetate and manganese chloride were used to modify HKUST-1 (Mn(N)/HKUST$1, \mathrm{Mn}(\mathrm{Ac}) / \mathrm{HKUST}-1$ and $\mathrm{Mn}(\mathrm{Cl}) / \mathrm{HKUST}-1)$ respectively, and the results were displayed in Fig. 2 (b). The mercury removal efficiency of unmodified HKUST-1 increased with the increase of temperature, reaching its maximum value of $31.15 \%$ at $175{ }^{\circ} \mathrm{C}$, and then remained basically flat between $20-30 \%$. The mercury removal efficiency of the samples modified by different manganese precursors was higher than that of HKUST-1 during 30-300 ${ }^{\circ} \mathrm{C}$. Among different kinds of manganese precursor, HKUST1 modified by $\mathrm{MnCl}_{2}$ shows the highest removal efficiency, up to $95 \%$ in the range of $250-300{ }^{\circ} \mathrm{C}$, which could be attributed to the combined effect of $\mathrm{Cu}, \mathrm{Mn}$ and $\mathrm{Cl}$.
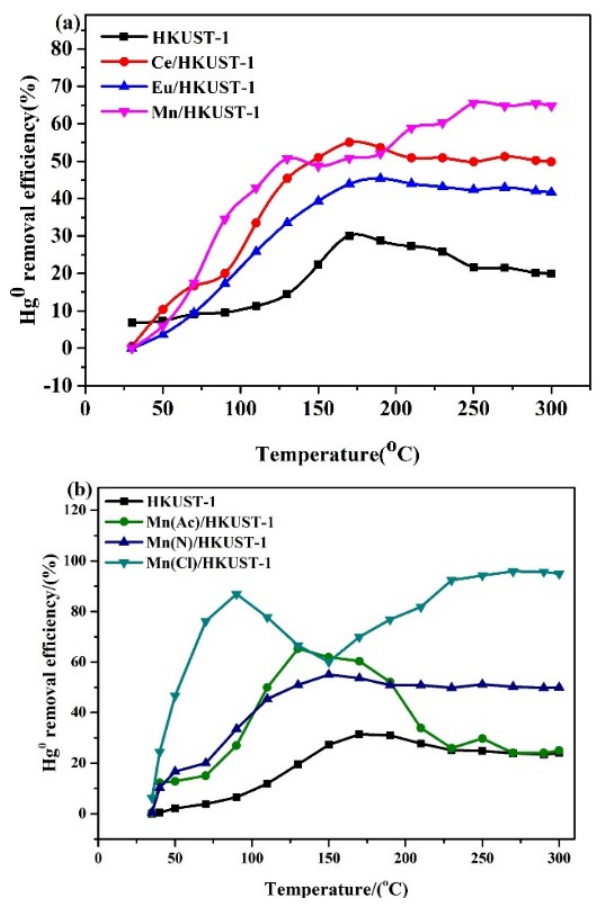

Figure 2 (a)Test of different metal modified HKUST-1 (b)Different manganese Mn precursors modification on

HKUST-1 test results (Reaction condition: $\mathrm{Hg}^{0}=30$ $\mu \mathrm{g} / \mathrm{m}^{3}, \mathrm{GHSV}=1.74 \times 10^{5} \mathrm{~h}^{-1}, \mathrm{~T}=30-300{ }^{\circ} \mathrm{C}$ )

\subsection{Characterization of samples}

\subsubsection{XRD analysis}

The XRD patterns reveal the crystallographic structures of the catalysts, as shown in Fig. 3. The pattern of HKUST-1 matches well with the simulated one, suggesting the material was prepared successfully with high purity [11]. There were still intact characteristic peaks of HKUST-1 after loading metals, indicating that the main structure was not affected by the impregnation loaded metal salt. In addition, there were no diffraction peaks of metal salts shown in the other three patterns. This means that $\mathrm{Ce}, \mathrm{Eu}$ and $\mathrm{Mn}$ were well distributed and stayed as amorphous. The uniform dispersion of metal element was beneficial to $\mathrm{Hg}^{0}$ oxidation [15]. The low crystallinities of the modified materials were conducive to the formation of surface defects of the samples and thus providing more reaction sites [16]. Therefore, it was believed that metal modified HKUST-1 can remove $\mathrm{Hg}^{0}$ much more efficient compared to pristine HKUST-1 [17].

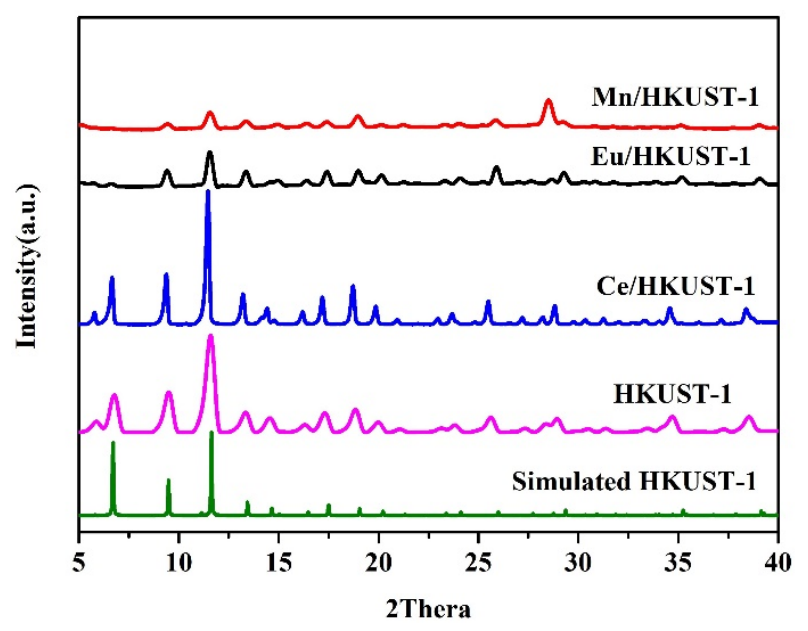

Figure 3 XRD pattern analysis

\subsection{2 $\mathrm{H}_{2}$-TPR experiment}

It was reported that there was a close correlation between the reduction capacity and catalytic property of the catalyst [18]. Therefore, the $\mathrm{H}_{2}$-TPR tests were conducted for four different metal-modified HKUST-1 samples to analyse their relative reducibility. As shown in Fig. 4, these four samples have peaks at different temperatures. Some distinctive peaks were displayed on the pattern. It can be found that two peaks appeared on the sample modified by $\mathrm{Mn}$, at the temperature of $374{ }^{\circ} \mathrm{C}$ and $453^{\circ} \mathrm{C}$, which corresponded to the reduction process of $\mathrm{Cu}^{2+}$ to $\mathrm{Cu}^{+}$and $\mathrm{Mn}^{4+}$ to $\mathrm{Mn}^{3+}$, respectively $[19,20]$. Compared with pristine HKUST-1, the reduction temperature of $\mathrm{Cu}^{2+}$ was lower, indicating Mn/HKUST-1 has more oxygen species to improve catalytic oxidation performance. Correspondingly, Mn/HKUST-1 showed higher catalytic capacity than the other three samples due to the lower reduction temperature was favourable for higher catalytic performance [21]. Therefore, the $\mathrm{H}_{2}$-TPR results gave explanation for the highest $\mathrm{Hg}^{0}$ removal efficiency of Mn/HKUST-1 among the four samples. 


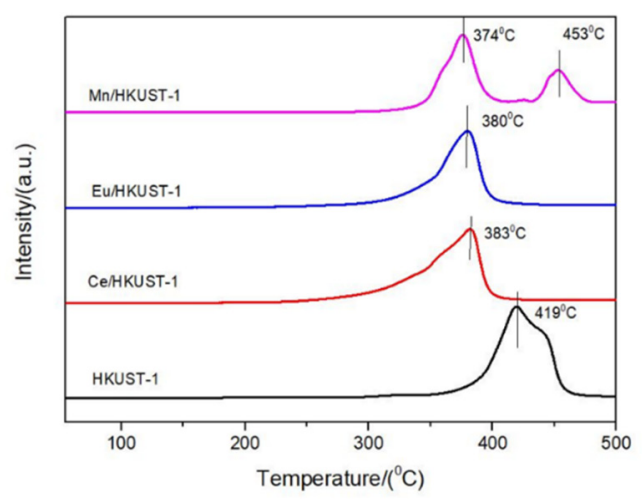

Figure $4 \mathrm{H}_{2}$-TPR test

\subsubsection{Thermogravimetric analysis}

Thermal stabilities of prepared materials were identified by TGA (shown in Fig. 5(a)). Pristine HKUST-1 and modified HKUST-1 experienced similar processes. The first major mass loss before $120{ }^{\circ} \mathrm{C}$ was ascribed to the water molecules removal which were physically adsorbed. The second large mass decline after $300^{\circ} \mathrm{C}$ was probably due to the decomposition of the HKUST-1 structure [22]. As a whole, HKUST-1 and modified HKUST-1 can keep the stable structure at a relatively high temperature.

The additional weight loss stages of modified HKUST1 compared to the pristine one was resulted from the decomposition of metal salts when temperature went higher than $350^{\circ} \mathrm{C}$. This was in agreement with the TGA curves for different metal salts as shown in Fig. 5(a).

It can be found that HKUST-1 modified by $\mathrm{MnCl}_{2}$ kept the last stable state at the highest temperature among three modified samples, indicating possessing the highest thermal stability, which is beneficial for $\mathrm{Hg}^{0}$ removal.

\subsubsection{In situ DRIFTS}

It was reported that $\mathrm{Hg}^{0}$ could combine with acid gas to form acid sites on the catalyst surface [23]. Therefore, in situ DRIFTS test was carried out to further study the active centres, the results were shown in Fig. 3(b). Some peaks displayed in the range of $1200-1700 \mathrm{~cm}^{-1}$. Two peaks centred at $1260 \mathrm{~cm}^{-1}$ and $1610 \mathrm{~cm}^{-1}$ were attributed to the Lewis acid sites, and the bands at $1450 \mathrm{~cm}^{-1}$ and $1670 \mathrm{~cm}^{-}$ ${ }^{1}$ were Brønsted acid sites [24]. The results showed that the largest amount of Brønsted acid sites presented on $\mathrm{Mn}(\mathrm{Cl}) / \mathrm{HKUST}-1$ surface, which resulted in the excellent removal performance of $\mathrm{Hg}^{0}$. Fig. 3(c) shows the relative amount of Lewis acid sites and Brønsted acid sites corresponding to Fig.3(b). Papers have illustrated that Brønsted acid sites were prior to Lewis sites for $\mathrm{Hg}^{0}$ removal [25]. Hence, the in situ DRIFTS test also supported the result of the highest $\mathrm{Hg}^{0}$ removal performance of $\mathrm{Mn}(\mathrm{Cl}) / \mathrm{HKUST}-1$.
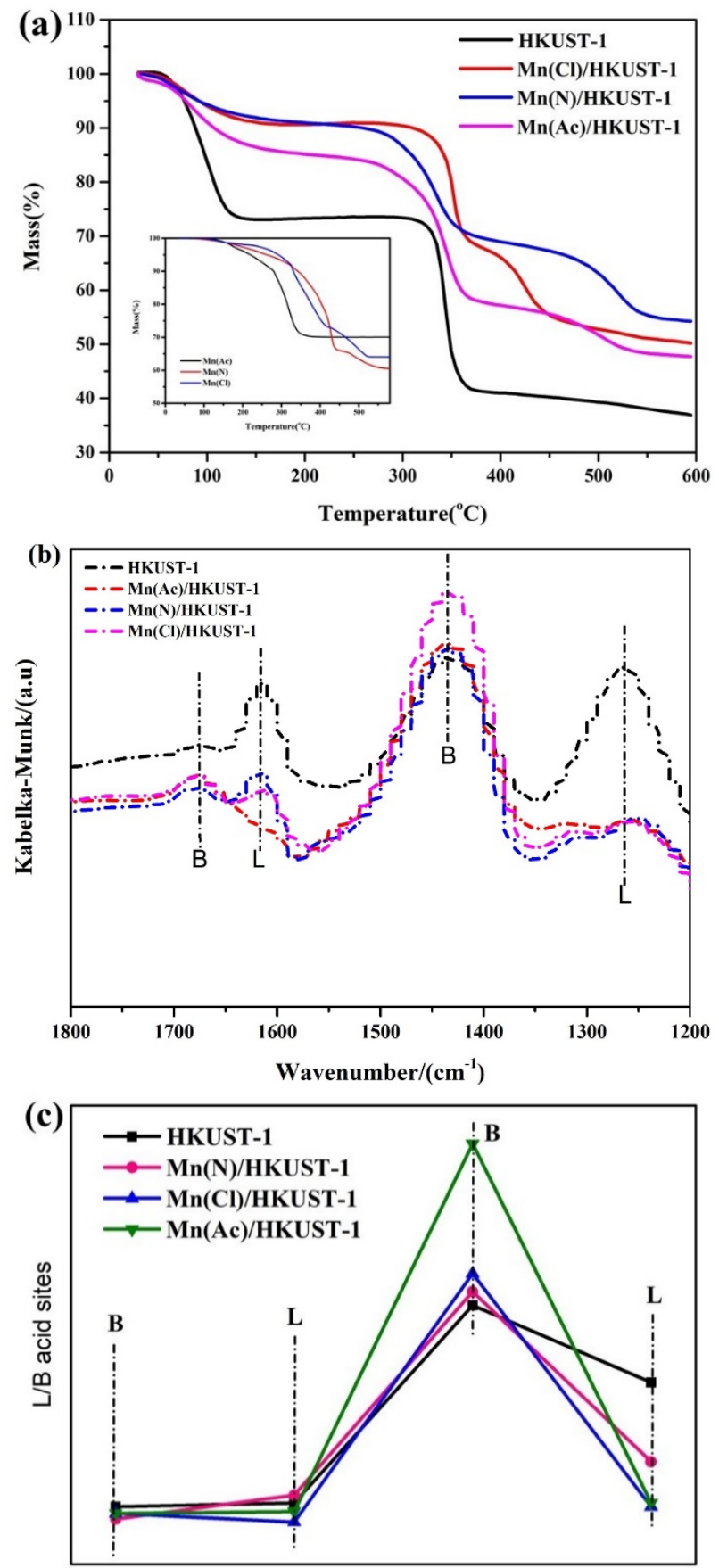

Figure 5 (a) Thermal stability test (b) In-situ DRIFT experiment (c) B/L acid sites ratio corresponding to (b)

\subsection{The effect of $\mathrm{SO}_{2}$ on $\mathrm{Mn}(\mathrm{Cl}) / \mathrm{HKUST}-1$}

It is generally reported that $\mathrm{SO}_{2}$ causes an inhibition of $\mathrm{Hg}^{0}$ removal [26]. Therefore, it is vital to study the $\mathrm{SO}_{2}$ effect on the sample performance, since $\mathrm{SO}_{2}$ normally exists in the flue gas. $\mathrm{MnCl}_{2}$ modified HKUST-1, as the optimal $\mathrm{Hg}^{0}$ removal material obtained in this work were taken as the sample to investigate $\mathrm{SO}_{2}$ effect on it. A typical temperature of $250^{\circ} \mathrm{C}$ was selected to study $\mathrm{SO}_{2}$ effect, since $\mathrm{Hg}^{0}$ removal efficiency reached the highest at this temperature. The efficiencies under three different gas conditions were investigated and the results are displayed in Fig. 6. 
Under the first condition, the fresh catalyst was heated to $250{ }^{\circ} \mathrm{C}$ in $\mathrm{N}_{2}$ for two hours. During this period, $\mathrm{Hg}^{0}$ removal rate was found to be maintained at about $70 \%$. Then it can be observed that the removal rate of $\mathrm{Hg}^{0}$ gradually decreased after $100 \mathrm{ppm} \mathrm{SO}_{2}$ was added into the gas at $120 \mathrm{~min}$, dropping by $30 \%$ within two hours. It can be speculated that this phenomenon was caused by the competitive adsorption of $\mathrm{SO}_{2}$ and $\mathrm{Hg}^{0}$ on the surface of the sample. It was also reported that $\mathrm{Hg}^{0}$ would compete adsorption with $\mathrm{SO}_{2}$ in the literature [27].

For the second condition, $5 \%$ oxygen was added and it was found that the mercury removal efficiency could rise to about $95 \%$. The above activity tests proved that a certain amount of $\mathrm{O}_{2}$ could enhance the $\mathrm{Hg}^{0}$ removal efficiency. After stabilizing for two hours, 100 ppm $\mathrm{SO}_{2}$ was also introduced. It was found that the removal efficiency of mercury still kept stable in the following two hours. This phenomenon indicated that $\mathrm{O}_{2}$ could not only promote the removal of $\mathrm{Hg}^{0}$, but reduce the competitive adsorption of $\mathrm{SO}_{2}$ [28]. It has been reported that part of $\mathrm{SO}_{2}$ was converted to $\mathrm{SO}_{3}$ in the presence of $\mathrm{O}_{2}$, which promotes the catalytic oxidation of $\mathrm{Hg}^{0}$ to some extent, thus reducing the toxicity of $\mathrm{SO}_{2}$ [29].

In order to further study $\mathrm{Hg}^{0}$ removal mechanism of $\mathrm{Mn}(\mathrm{Cl}) / \mathrm{HKUST}-1$ with the presence of $\mathrm{SO}_{2}$, the experiment under the third condition was carried out. The only difference between the second condition was that the fresh sample was pre-treated by $100 \mathrm{ppm} \mathrm{SO}_{2}$ for 2 hours at room temperature before $\mathrm{Hg}^{0}$ removal test. As can be seen from the figure, the removal efficiency kept the same as the second condition during the first $120 \mathrm{~min}$. However, the removal efficiency began to decrease afterwards. This phenomenon may due to the presence of some fresh Mnbased active sites on the surface of the pre-treated sample, which promotes the removal of $\mathrm{Hg}^{0}$. While Mn-based active sites were gradually poisoned with the continuous $\mathrm{SO}_{2}$ injection. It is interested that the removal rate still reached around $60 \%$, the reason was inferred to the new active sites generation on the surface to facilitate the removal of $\mathrm{Hg}^{0}$.

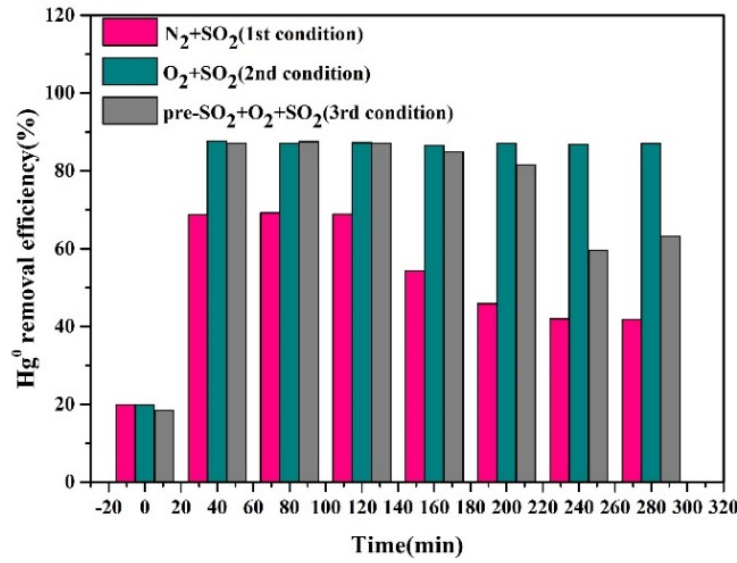

Figure $6 \mathrm{SO}_{2}$ effect on $\mathrm{Mn}(\mathrm{Cl}) / \mathrm{HKUST}-1$ at $250^{\circ} \mathrm{C}$

\section{Conclusion}

In conclusion, HKUST-1 and modified HKUST-1 were studied for $\mathrm{Hg}^{0}$ removal. Among these, $\mathrm{Mn}(\mathrm{Cl}) / \mathrm{HKUST}-1$ showed the highest $\mathrm{Hg}^{0}$ removal efficiency of $95 \%$, which is competitive compared to most reported materials. The In-situ DRIFT result indicated that $\mathrm{MnCl}_{2}$ promotes Brønsted acid sites generation, thus providing the beneficial factor for $\mathrm{Hg}^{0}$ removal. It is found that $\mathrm{Mn}$ distributed evenly on the HKUST-1 from the XRD result, which is favourable for $\mathrm{Hg}^{0}$ removal. Moreover, $\mathrm{Mn}(\mathrm{Cl}) / \mathrm{HKUST}-1$ showed a good $\mathrm{SO}_{2}$-resistance in the presence of $\mathrm{O}_{2}$. Based on the screening results in this study, $\mathrm{Mn}(\mathrm{Cl}) / \mathrm{HKUST}-1$ was proved to be a potential $\mathrm{Hg}^{0}$ removal material. Further studies on the mechanism of $\mathrm{Hg}^{0}$ removal by $\mathrm{Mn}(\mathrm{Cl}) / \mathrm{HKUST}-1$ will be carried out in the future progress.

\section{Acknowledgements}

Following funding bodies are acknowledged for sponsoring this research: National Key R\&D Program of China (2017YFB0603202), Ningbo 'Science and Technology Innovation 2025' Major Project (2018B10091) and Zhejiang Provincial Key Laboratory Programme (2020E10018).

\section{Reference}

1. Wang, S., et al., Atmospheric Chemistry and Physics, 2010. 10(3): p. 1183-1192.

2. Emission standards for air pollutants from thermal power plants [S] GB13223. 2011.

3. Zhao, S., et al., Fuel, 2015. 158: p. 891-897.

4. Presto, A.A. and E.J. Granite, Environmental science \& technology, 2006. 40(18): p. 5601-5609.

5. Fang, G.C., et al., Environ Monit Assess, 2011. 181(14): p. 273-89.

6. Liu, Y., H. Li, and J. Liu, Fuel, 2016. 184.

7. Xiao, Z., et al., Journal of hazardous materials, 2016. 320.

8. Chiericatti,C.,et al,,Mecroporous and mesoporous materials,2012. 162 :p.60-63

9. Chen, D., et al., Fuel, 2018. 217: p. 297-305.

10. Kim, Y.J., et al., App Cat B :Enviromental, 2012. 126

11. Chen, Y., et al., Progress in Natural Science: Materials International, 2018. 28(5): p. 584-589.

12. Li, J., et al., Science Robotics, 2018. 3(19).

13. Yang,G.,etal.,App Cata B: Environmental, 2019. 245

14. Zhang, A., et al., Chemical Engineering Journal, 2014. 236: p. 29-38.

15. Li, H.H., et al., Chem.Pap, (2017) 71 :1569-1578

16. Criado, M., A. Fernández-Jiménez, and A. Palomo, Microporous and Mesoporous Materials, 2007. 106(1-3): p. 180-191.

17. Li, H., et al., Chemical Papers, 2017. 71(9): p. 15691578.

18. Torre-Abreu, C., et al., Applied Catalysis B: Environmental, 1997. 14(3-4): p. 261-272.

19. Zhang, X., et al., Chemical Engineering Journal, 2017. 326: p. 551-560. 
20. Wan, Y., et al., Jour of Cata, 2004. 227(1): p. 242-252.

21. Zhang, X., et al., Molecular Catalysis, 2020. 482: p. 110701.

22. $\mathrm{Mu}, \mathrm{X}$., et al., Microporous and Mesoporous Materials, 2018. 270: p. 249-257.

23. Wilcox, J., et al., International Journal of Coal Geology, 2012. 90-91: p. 4-20.

24. Lin, S.D., A.C. Gluhoi, and B.E. Nieuwenhuys, Catalysis Today, 2004. 90(1-2): p. 3-14.

25. Zhao, H., et al., Environmental science \& technology, 2016. 50(2): p. 1056-1064.

26. Chen, Y., et al., Fuel, 2021. 285: p. 119064.

27. Li, H., et al., Fuel, 2020. 260: p. 116289.

28. Li, J., et al., Environ Sci \& technology, 2010. 44(1): p. 426-431.

29. Li, H., et al., Fuel, 2017. 208: p. 576-586. 